\title{
Acoustic Transmission Technology in Production Logging and Application
}

BaoHui ZHANG ${ }^{1^{*}}$, YanSong WANG ${ }^{1}$, HongGang WEI ${ }^{1}$, ShiTong WU ${ }^{1}$, Yun LI ${ }^{1}$, YiMing WANG ${ }^{1}$, ShaoFei GUO ${ }^{2}$, Jun $\mathrm{FU}^{3}$, ZhanSheng $\mathrm{LI}^{1}$, Cheng $\mathrm{HUANG}^{1}$, Yan $\mathrm{GAO}^{2}$, JianJie NING ${ }^{1}$, HongYuan SUN ${ }^{1}$, Hua ZHAO ${ }^{1}$

${ }^{1}$ Petroleum production engineering research institude of huabei oilfield company, Renqiu, 062552, China.

${ }^{2}$ The first exploit factory of huabei oilfield company, Renqiu, 062552, China.

${ }^{3}$ The erlian filiale of huabei oilfield company, xilinhaote, 026000, China.

ABSTRACT: In this paper, we investigate an acoustic transmission technology in Production Logging, The subsurface equipment has be installed during pump operation, the logging tools and transmission system Installed in the bottom of the pump and into the wellbore together. Raw log datas are transformed into regular coding acoustic signals that are transmitted to the wellhead along the tubing and are received by acoustic detector, the signals are recorded by a computer. Engineers analyze and interpret these signals to get the dynamic production data of the well under the current production status. It can realize the well long-term monitoring. The technology was applied in Huabei Oilfield and the transmission depth is close to 2000m. The technology can provide accurate information for development plan of oilfield. This paper also discusses the attenuation factor of acoustic in the tubing and the design principle of the technology.

KEYWORDS: Oil well; Acoustic wave; Transmission; Logging; Encode; Technology

\section{INTRODUCTION}

With further oilfield development, production logging technology shows the special function, for example, It provide various data for reservoir engineering and production engineering or Check the effect of well stimulation. Some conventional production logging methods cannot be applied due to the influence of many factors, such as the well condition, so the oil well dynamic production data is too lack to meet the needs of the reservoir comprehensive treatment. Acoustic transmission technology can solve logging problems for special oil well.

\section{TECHNOLOGY PRINCIPLE}

The acoustic transmission system and logging instrument Installed in the bottom of the pump and into the wellbore together during pump operation, they consecutive working long hours in the wellbore till pump inspection next time. Logging instruments are working according to set procedures in the well, log data is converted to binary code sound waves through the equipment. The wave is transmitted to the ground along the tubing and be received and recorded at well head. Engineers can get downhole dynamic data after computer interpretation.

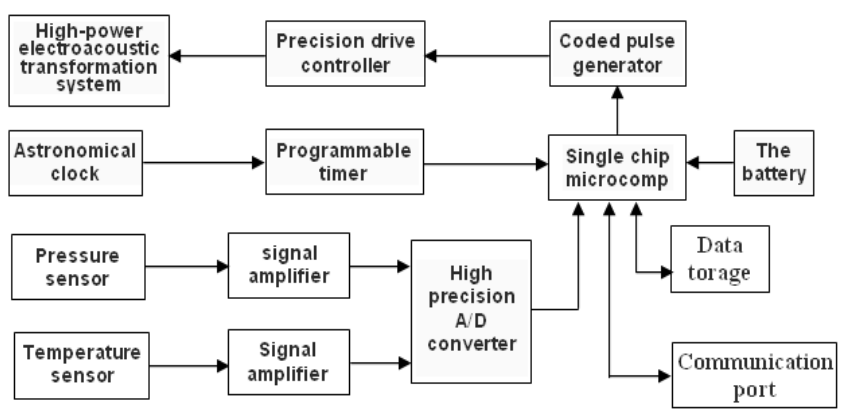

Figure 1 Process principle diagram

\subsection{The electroacoustic transducer}

Sound wave transmission is the key to the technology, so electroacoustic transducer is the core component of downhole tools. It must meet the following conditions in the design: (1)High output power. (2)Far transmit distance. (3)the single launch time is short. (4)Sonic pulse is easy to distinguish. (5)Able to work for a long time. We achieve the goal by using a strong electromagnet to drive a iron core.

\subsection{Coding scheme}

How to encode log data to form the pulse signal? it Is big key in the software program of the technology. Encoding rules is need to meet the conditions: (1) Suitable for sound waves transmit in tubing. (2) Accurate, get the error free encode. (3)Convenient, easy to code In the program. (4)Reduce the workload 
of electroacoustic transducer as far as possible to achieve the purpose of saving energy. (5)Satisfy the precision of the data.

\subsection{The receiving device}

The main frequency of the signal wave in tubing string fall in between $1500 \mathrm{~Hz}$ to $3000 \mathrm{~Hz}$, we have designed a high sensitive ground receiving device based on the principle of resonance. The natural oscillation frequency of the receiver equal to the frequency of the signal in the tubing. The effect of the acoustic signals is greatly enhanced because of resonance effect. The clarity of signals meet the needs of the interpretation.

\subsection{Anti-jamming technique}

There may be influencing factors during the reception, such as sound of the wind v machine noise 、 conversation v the water sound, and so on. The acoustic signal is difficult to identify and explain. Therefore, we designed a anti-jamming technique to filter out interference signal and keep useful signal, sample signal was processed through spreading and amplifying and it was displayed to generate the correct interpretation data. The basic principle is as follows: First of all, remove the noise signals according to the characteristics of the transmission signal frequency, then we perform noise reduction processing according to the audio properties of noise. This method can achieve very good effect to remove most of the noise.

\section{ATTENUATION OF SOUND DURING WELL}

We experiment with signal transmission in different depth of downhole respectively (5 different Wells, $500 \mathrm{~m} 、 1100 \mathrm{~m} 、 1240 \mathrm{~m} 、 1540 \mathrm{~m}$ and $1940 \mathrm{~m})$. We analyzed the acoustic amplitude under different depth, regular results have been gained. The relationship between acoustic signal intensity and transmission depth conform to the law of exponential decay. As shown in figure 2 .

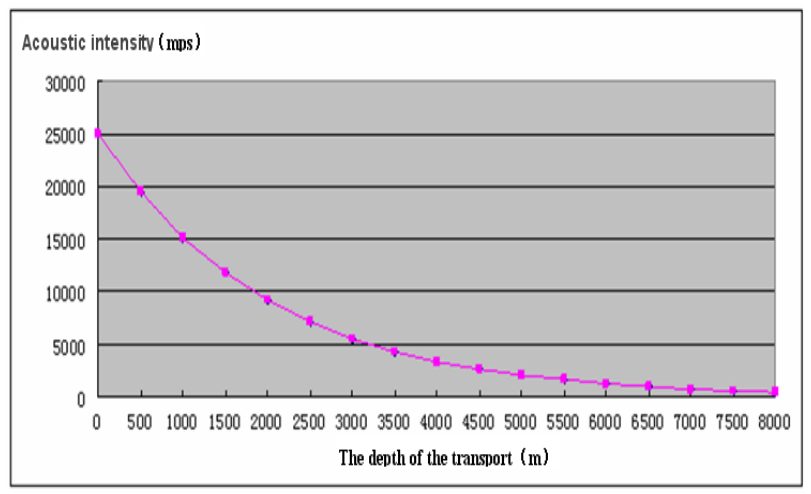

Figure 2 The relationship between acoustic intensity and the depth of the transport
In general, signal resolution will be difficult when signal amplitude is less than $2300 \mathrm{mps}$, signal amplitude is 9200 mps when the transmission depth is $1940 \mathrm{~m}$, the amplitude is much higher than the bottom line $(2300 \mathrm{mps})$ and the signal can distinguish and explained very well. So the technology has practical application value within $2000 \mathrm{~m}$ in the well. From the curve, the corresponding depth of the transport is $4750 \mathrm{~m}$ when the signal amplitude reduced to $2300 \mathrm{mps}$, acoustic signals can be used as information carrier within $3000 \mathrm{~m}$ of depth might even be a conservative estimate.

\section{FIELD APPLICATION}

This technology is applied in 5 wells in Huabei oilfield, the maximum depth of signal transmission is $1941.52 \mathrm{~m}$. We heard the clear sound if we listened at the wellhead when the signal launch in the well, The method was proved feasible by the experiment that treat production string as information transmission medium, this technology has practical value in the actual logging work.

The figure below shows production logging curve of oil well in Huabei oilfield, although the application of time is not long, but the curve display the variation of temperature and pressure of the well from the instrument is run into the wellbore to the well recover it's production, from the well stop producing to take out instrument next time. This is a prime example.

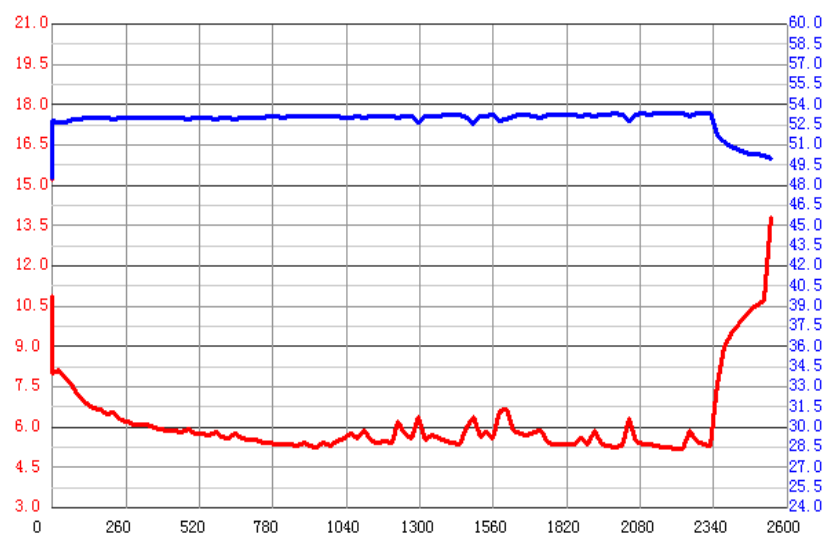

Figure 3 logging curves.

\section{ECONOMIC EFFICIENCY}

Using this technology, the downhole logging instrument was brought into hole with the pump, not need an extra operation, and not need the logging team, can save a lot of logging fee. Compared with the conventional logging method, it can save 100000 RMB per well. 


\section{CONCLUSION}

(1) In general, use cable to transmit signals in well logging is necessary. Acoustic transmission technology is a breakthrough, the downhole tool can transmit log data to the ground by binary encoding acoustic signals through the tubing string, meet the needs of the nonwireline logging method.

(2) Receiving probe can be very small to make them easier to be used in the oil production plant, it may adsorbs on flange、 stuffing box , polished rod through the magnetic force.

(3) There is a significant difference between the waveform of signal and ambient noise that appears on a computer screen, so it is difference between their sound effects. Engineers can obtain interpretation results according to the differences and the anti-interference technology.

(4) It provides technological basis for expanding to other fields, we developed a new technology to control downhole tool by transmitting acoustic signal to the underground along tubing-casing annular space, we got success under the the depth of $1257 \mathrm{~m}$.

\section{REFERENCES}

[1] Zhang Jianjun and Zhang Baohui: Well Testing, Vol. 16, No.3 (2007), p. 73

[2] Liu Xuanchao: Petroleum Instruments, Vol. 20, No.5 (2006), p. 28 